\title{
PRELIMINARY STUDY OF MULTI-VIEW IMAGING FOR ACCURATE 3D RECONSTRUCTION USING STRUCTURED LIGHT SCANNER
}

\author{
Siti Syazalina Mohd Sobani ${ }^{1}$, Siti Asmah Daud ${ }^{2}$, Nasrul Humaimi Mahmood ${ }^{3}$ \\ ${ }^{1,2}$ Postgraduate Student, ${ }^{3}$ Senior Lecturer, Faculty of Biosciences \& Medical Engineering, Universiti Teknologi \\ Malaysia,Johor, Malaysia,syazalina.sobani@yahoo.com, sitiasmahdaud@gmail.com,nasrul@fke.utm.my
}

\begin{abstract}
This paper presents a multi-view structured-light approach for surface scanning to reconstruct three-dimensional (3D) object using a turntable. It is a modification from DAVID 3D Scanner SLS-1 (Structured-Light Scanner) as a starting point of study on improving and builds a complete system of $3 D$ structured-light based scanner. This type of scanner uses a video projector to project various patterns onto an object which is going to be digitized or reconstruct to a $3 D$ model. At the same time, a camera will record and capture the scene at least one image of each pattern from a certain point of view for example from right, left, above or below of the video projector. Then, 3D meshes of surface of the object will be computed based on the deformations of the projected patterns. The preliminary results show that object which are model of prostheses are successfully reconstructed.
\end{abstract}

Index Terms: 3D scanner, structured-light scanner, 3D reconstruction, and multiple-view

\section{INTRODUCTION}

Structured-light three-dimensional (3D) scanning technique is a non-contact active measurement method. It is a system of a fixed scanner with a turntable which can scan surfaces of object fast as many time as necessary to get sets of point clouds needed to reconstruct 3D model of the object scanned [1-2]. 3D scanners particularly being used to digitized 3D surface in $3 \mathrm{D}$ entertainment industry, robotic vision, digital model of heritage sites and historical building preservation (for archaeologists and curators) and also medical field (for orthotics and prosthetics) [2-5]. The system has many advantages such as simple optical arrangement, stable and also fast acquisition speed. The structured-light sensors use many light patterns in order to acquire accurate measurement and information as high as possible. The light patterns are usually consisted of grids, gratings and stripes. Fig -1 shows some examples of structured-light patterns.

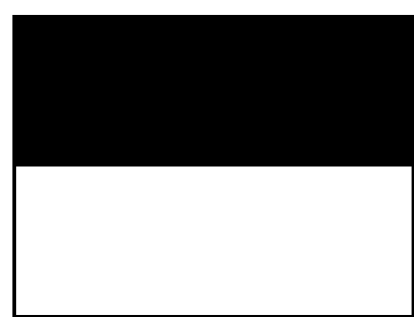

(a)

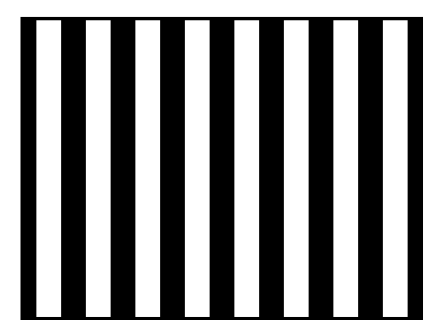

(b)

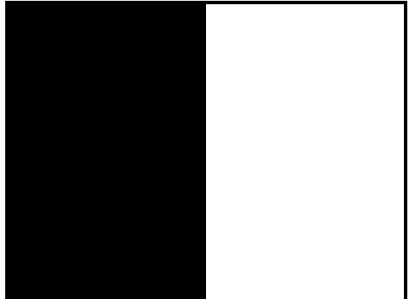

(c)

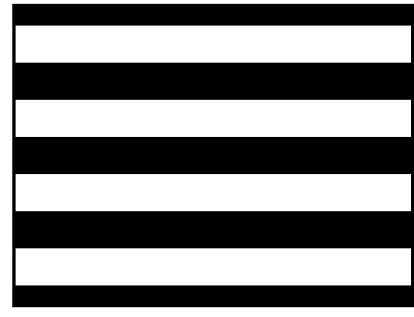

(d)
Fig -1: Examples of structured-light patterns

Within past decades, many researches have been generated by $3 \mathrm{D}$ object modeling and it is a constraint in computer vision field. It is encouraged to use this technology to digitized 3D surfaces as it did not need a direct contact which can affect the accuracy of reconstructed model. Cheng et al. improved the resolution and accuracy for spatial neighborhood coding by $46 \%$. This is also has fault tolerance ability using groups of color coded pattern [6]. Keerativittayanun et al. presented 3D reconstruction using primary colours structured-light which are red, green and blue. There are 108 stripes in each separate 10 pixels width pattern [7]. Kazó et al. provided automated scanning and turntable system which reconstruct high-quality realistic 3D models [8]. Zhou et al. recently presented realtime reconstruction with $300 \mathrm{fps}$ high-resolution point cloud registration using structured-light illumination (SLI) 3D scanner and also contribute to research community with 3D gesture and face databases [9]. 


\section{METHODOLOGY}

Most 3D scanners (including the scanner which will be described in this paper) can do the scanning from a single viewpoint only at one time. So it is a need to scan one by one, angle by angle to get more information in order to reconstruct a complete 3D model. In this paper, it is proposed that overall processes are user friendly and required minimal preparation of the experimental environment while the reconstruction is done using DAVID Laser Scanner software.

The setup of the experimental is shown in Fig -2 where the projector displayed structured-light patterns on a calibration board which is half-folded with an angel of $90^{\circ}$. Camera is placed behind and slightly higher than the projector to record and capture the scene clearly. Fig-3 shows an object is placed on a turntable in front of calibration board while Fig-4 shows two examples of objects used in the test which are Object 1 and Object2. The processes to reconstruct 3D model from an object are consisted of two phases which explained as follows.

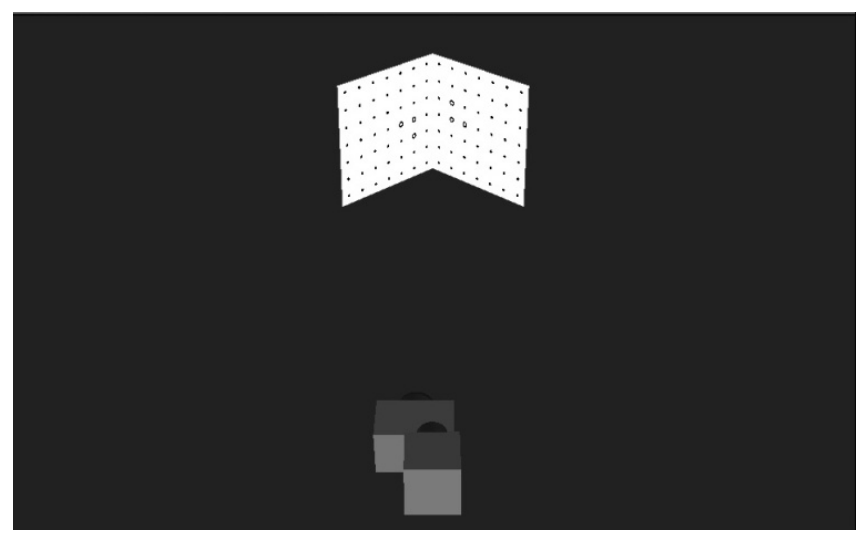

Fig -2: Experimental set up

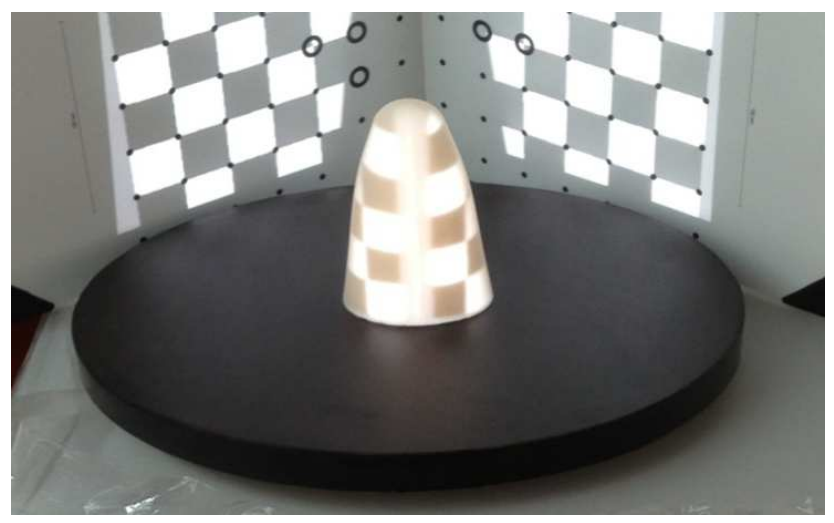

Fig -3: Object placed on turntable in front of calibration board

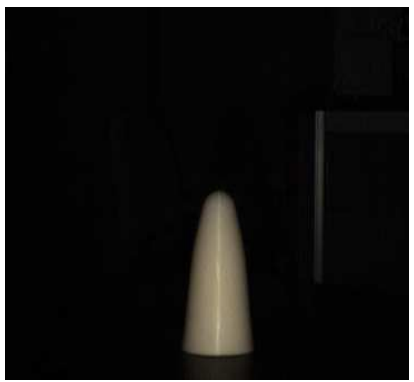

(a)

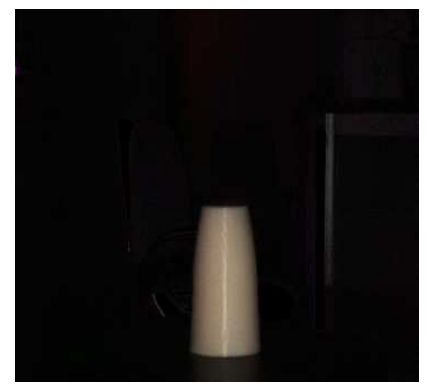

(b)
Fig -4: (a) Object1 and (b) Object2 that has been used to test the system

\subsection{Camera Calibration}

The most important thing to do in $3 \mathrm{D}$ reconstruction from corresponding two-dimensional (2D) images, one must know how to obtain unknown parameters of camera model [10]. This process is well-known as camera calibration in computer vision field. Basically, camera calibration can be categorized into two main groups which are non-self (photogrammetric) calibration and self-calibration [11]. At present, various methods are listed that can be reviewed such as direct linear transformation method, nonlinear optimization method, twostep method, biplane method, self-calibration method and plane pattern method (which has been on focus by many researchers).However, all these camera calibration techniques do not work when the charge-couple device (CCD) plane is tilted but parallel to the lens plane [1]. Structured-light scanner needs both projector and camera to be calibrated. The calibration is done based on the well-known pinhole camera model as shown in Fig-5, where 0 is the optical centre, the projection of real world point $\left[\begin{array}{lll}X & Y & Z\end{array}\right]^{\mathrm{T}}$ onto the image plane at projection of pixel number $\left[\begin{array}{ll}u & v\end{array}\right]^{\mathrm{T}} \mathrm{u}=\frac{\mathrm{Xf}}{\mathrm{Z}}$ and $\mathrm{v}=\frac{\mathrm{Yf}}{\mathrm{Z}}$, noted that $\mathrm{f}$ is the focal length. This relation then reformulated using projective geometry framework and expressed as $\lambda\left(\begin{array}{l}\mathrm{u} \\ \mathrm{v} \\ 1\end{array}\right)=\left(\begin{array}{cccc}\mathrm{f} & 0 & 0 & 0 \\ 0 & \mathrm{f} & 0 & 0 \\ 0 & 0 & 1 & 0\end{array}\right)\left(\begin{array}{l}\mathrm{X} \\ \mathrm{Y} \\ \mathrm{Z} \\ 1\end{array}\right)$ noted that the homogeneous scaling factor is $\lambda=\mathrm{Z}$. Then, this relation will be solved to find intrinsic and extrinsic parameters of the camera by considering the radial distortion and camera rotation and camera rotation involved. 


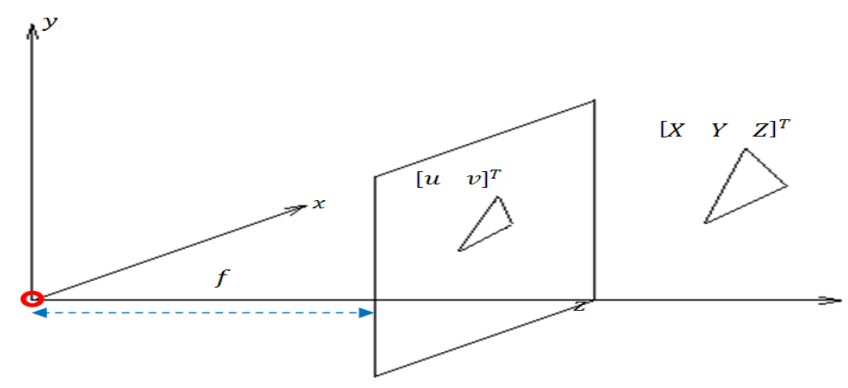

Fig -5: Pinhole camera model

\subsection{Point Cloud Registration}

Multi-view approach is used in order to acquire a complete model of object. This can help to overcome the limitation in camera 2D view, so the object can be scanned from different viewpoints. The viewpoints are according to specific angle that can be acquired by rotating the object to be scanned using a turntable while the projector and camera are fixed at a certain distance and place. Point cloud registration or also called as splicing is a process to convert more than one coordinate system into one coordinate system [12]. It works by aligning point clouds of the scanned surface one by one, where local coordinate is given to a point cloud from each scan surface according to the position of the object and scanner obtained via calibration of camera. In order to merge the point clouds, at least three pairs of corresponding points or $\mathrm{N} \ll 3$ to be ideal. This is essential so that when all the scans are merged together to form a complete 3D model, each view transformation is same to world coordinate. It is computed by considering the $\mathrm{N}$ pairs of corresponding 3D points which can be expressed as $\left\{\left(\mathrm{p}_{1} ; \mathrm{q}_{1}\right),\left(\mathrm{p}_{2} ; \mathrm{q}_{2}\right), \ldots,\left(\mathrm{p}_{\mathrm{N}} ; \mathrm{q}_{\mathrm{N}}\right)\right\}$, in order to find a rotation matrix, $\mathrm{R}$ and translation vector, $\mathrm{T}$ so that $R p_{j}+T=q_{j}$, where $j=1,2,3, \ldots, N$. There are manual and automatic point cloud registration algorithm presented in the $3 \mathrm{D}$ reconstruction. Manual registration is done by putting markers where is necessary onto the scanned surface. Compared to automatic registration which is much simpler to compute, however it is also known that it has limitation such as poor stability, insufficient accuracy, low speed, etc [2].

\section{RESULTS AND DISCUSSIONS}

It is important to have the latest working device to do the test to make sure it will run smoothly. DAVID 3D Scanner suggested to use a standards PC with Windows XP, Vista or 8 ( 32 or 64 bit), with two available USB ports and at least must have $2 \mathrm{GHz}$ CPU, 1 GB RAM, 3D graphics card for example NVIDIA GeForce or ATI Radeon. Considering the recommendations, the $\mathrm{PC}$ used for this project is consisting of $2.40 \mathrm{GHz}$ Intel Core i5 processor, NVIDIA GeForce with CUDA graphics card and 4GB RAM. Each scanning needs around 15 seconds to record a part of surface of an object. Fig-
6 shows examples for several scans of an object taken from different angle of views using turntable.

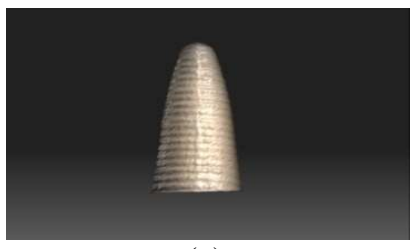

(a)

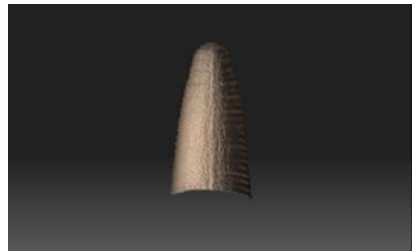

(c)

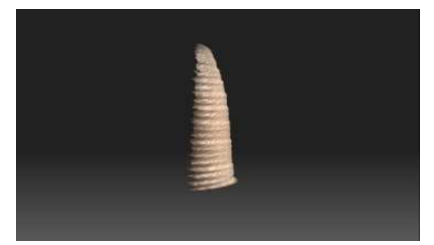

(b)

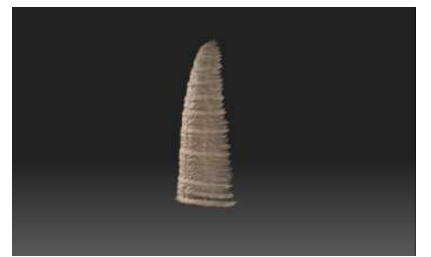

(d)
Fig -6: Different angles of scans for an object (a) $0^{\circ}$, (b) $90^{\circ}$, (c) $180^{\circ}$ and (d) $270^{\circ}$

This research does not focused on the time required to do a complete $3 \mathrm{D}$ reconstruction but the accuracy and quality of the $3 \mathrm{D}$ models. Fig-7shows the results of alignment or point clouds registrations while Fig -8shows the complete 3D models after shape fusion for the two tested objects which are Object1 and Object2 respectively. However, the 3D reconstruction result did not come out well since the surfaces scanned are not smooth and some parts of it are missing. So it affects the 3D model which there is some flat part at the corner. This is also an effect of the interference of light that causes the scanner cannot get all information required while scanning.

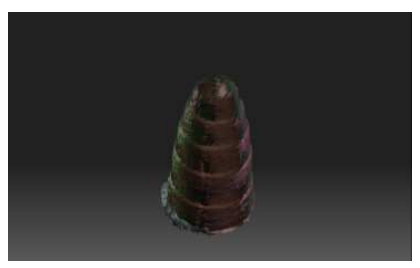

(a)

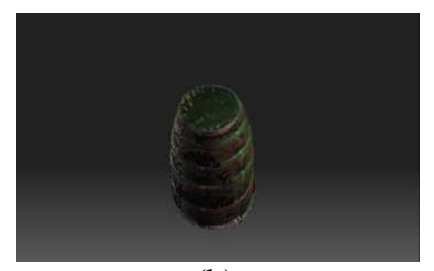

(b)
Fig -7: Aligned scans for (a) Object1, and (b) Object2

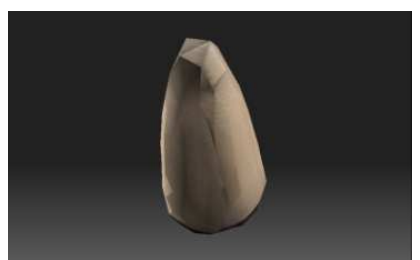

(a)

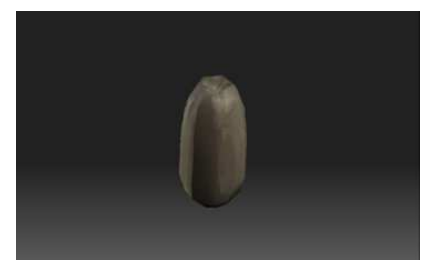

(b)
Fig -8: Complete 3D model for (a) Object1, and (b) Object2 


\section{CONCLUSIONS}

This paper proposes structured-light scanning approach for 3D reconstruction inspired by DAVID 3D Scanner SLS-1 (Structured-Light Scanner). It is a user friendly scanning method, so it can be used by anyone who is non-expert in this field of study. However, the interference of light affects the accuracy of $3 \mathrm{D}$ reconstruction. It is suggested to do the scan in a dark environment so the information that needed to be recorded is more accurate. In the future, there are some plans to improve the reconstruction so it will be higher resolution, accurate and more realistic.

\section{ACKNOWLEDGEMENTS}

The authors would like to express their gratitude to Ministry of Science Technology and Innovation (MOSTI) that provide eScience Fund research grant (Vote Number: 4S027) for this project and also scholarship from the Ministry of Higher Education of Malaysia. Thank you very much.

\section{REFERENCES}

[1] J. Li, Y. Guo, J. Zhu, X. Lin, Y. Xin, K. Duan, and Q. Tang, "Large depth-of-view portable three-dimensional laser scanner and its segmental calibration for robot vision," Optics and Lasers in Engineering, vol. 45, no. 11, pp. 1077-1087, Nov. 2007.

[2] J. Chen, X. Wu, M. Yu Wang, and X. Li, “3D shape modeling using a self-developed hand-held 3D laser scanner and an efficient HT-ICP point cloud registration algorithm," Optics \& Laser Technology, vol. 45, pp. 414-423, Feb. 2013.

[3]. B. W. He, Z. X. Long, and Y. F. Li, "The research of an automatic object reconstruction method based on limit visible region of the laser-scanning vision system," Robotics and Computer-Integrated Manufacturing, vol. 26, no. 6, pp. 711-719, Dec. 2010.

[4]. S. Al-kheder, Y. Al-shawabkeh, and N. Haala, "Developing a documentation system for desert palaces in Jordan using 3D laser scanning and digital photogrammetry," Journal of Archaeological Science, vol. 36, no. 2, pp. 537-546, Feb. 2009.

[5]. B. Coudrin, M. Devy, J.-J. Orteu, and L. Brèthes, "An innovative hand-held vision-based digitizing system for 3D modelling," Optics and Lasers in Engineering, vol. 49, no. 9-10, pp. 1168-1176, Sep. 2011.

[6]. F.-H. Cheng, C.-T. Lu, and Y.-S. Huang, "3D Object Scanning System by Coded Structured Light," 2010 Third International Symposium on Electronic Commerce and Security, pp. 213-217, Jul. 2010.

[7]. S. Keerativittayanun, T. Kondo, P. Sira-uksorn, T. Phatrapornant, and M. Sato, "3D scan of a color object using a color structured light pattern," 2011 IEEE 7th International Colloquium on Signal Processing and its Applications, pp. 460-463, Mar. 2011.
[8]. C. Kaz, "High-quality Structured-light Scanning of 3D Objects using Turntable," pp. 553-557, 2012.

[9]. Y. Zhou, K. Liu, J. Gao, K. E. Barner, and F. Kiamilev, "High-Speed Structured Light Scanning System And 3d Gestural Point Cloud Recognition University of Delaware , Newark, DE , USA , 19716 School of Electrical Engineering and Information , Sichuan University , China," no. 0812458, 2013.

[10] J. Dong and Z. Qin, "Camera self-calibration based on single rectangle," 2012 Third International Conference on Intelligent Control and Information Processing, pp. 674-677, Jul. 2012.

[11] S. Sugimoto and M. Okutomi, "Camera Self Calibration Based on Direct Image Alignment," no. Icpr, pp. 32403243, 2012.

[12] Z. Yi, G. Liwei, and Y. Li, "Research on error propagation of point cloud registration," 2012 IEEE International Conference on Computer Science and Automation Engineering (CSAE), no. 2, pp. 18-21, May 2012.

\section{BIOGRAPHIES}

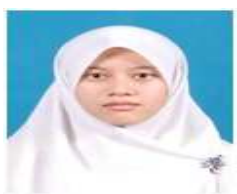

Siti Syazalina Mohd Sobani graduated from Universiti Teknologi Malaysia with Bachelor's Degree of Engineering (Electrical - Medical Electronics) in 2012.

She is currently a Ph.D student who undergoes research of image processing centered on 3D reconstruction.

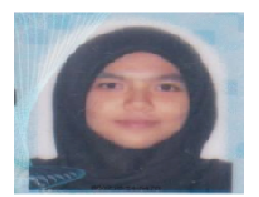

Siti Asmah Daud obtained her Bachelor's Degree of Engineering (Electrical Medical Electronics) from Universiti Teknologi Malaysia in 2012. She continues her study as a $\mathrm{Ph} . \mathrm{D}$ research student in exploring of infrared sensor in reconstructing a $3 \mathrm{D}$ image.

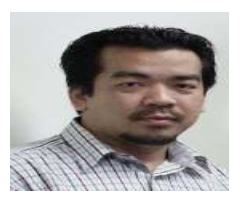

Nasrul Humaimi Mahmood obtained his PhD Degree from University of Warwick, United Kingdom. He is a Senior lecturer at Faculty of Electrical Engineering, Universiti Teknologi Malaysia. He currently served as a Head of Department at Faculty of Biosciences and Medical Engineering. 